\title{
The socioeconomic roots of shame and perceptions of social inadequacy
}

\author{
Lloyd Brandts ${ }^{1}$, Hans Bosma ${ }^{1}$, Audrey Simons ${ }^{1}$, Danielle Groffen ${ }^{1}$, \\ Marjan van den Akker ${ }^{2,3}$ \\ ${ }^{1}$ Maastricht University, Social Medicine, CAPHRI, Maastricht, The Netherlands, \\ ${ }^{2}$ Maastricht University, Family Medicine, CAPHRI, Maastricht, The Netherlands, \\ ${ }^{3}$ Catholic University Leuven, General Practice, Leuven, Belgium
}

\begin{abstract}
Introduction. Cumulating reports on the adverse health effects of income inequality hypothesise on underlying processes related to health compromising, negative social comparisons in people with a low socioeconomic status (compared to those who are socioeconomically better-off). As this hypothesis of "internalized inferiority" has not yet been examined explicitly, we set out to examine whether internalized inferiority is indeed more common in low socioeconomic status groups.

Method. Dutch SMILE data on 1,477 participants, aged 58-94 in 2008 were used. Income in adulthood (measured several times between 2002 and 2008), education in adulthood (measured several times between 2002 and 2008), education of parents (measured in 2005), and poverty in childhood (measured in 2004) were related to general shame (measured in 2009) and social inadequacy (measured in 2004 and 2008), using logistic regression analyses.

Results. Both education and income-related socioeconomic measures from childhood and adulthood had independent associations with social inadequacy. Poverty in childhood was related to reports of general shame.

Discussion.In this cohort the socioeconomic status of middle-aged and older men and women, especially its financial component, impacts cognitions and feelings of internalized inferiority. Childhood socioeconomic circumstances, particularly experiences of poverty, contributed independently. Our findings suggest that negative social comparisons and internalized inferiority might be possible key players in the association between low socioeconomic status and poor health.
\end{abstract}




\section{Keywords}

Socioeconomic status; internalised inferiority; social inadequacy; adulthood; childhood.

\section{Introduction}

Researchers have been trying to find the epidemiological key that underlies the globally occurring socioeconomic inequalities in health. Growing evidence points to processes related to social comparison being the underlying cause for health differences between social classes (Wilkinson, 2006). According to this hypothesis, the material reality of inequality is somehow embedded in people's image of themselves as being higher or lower in the societal pecking order. Cumulative evidence supports the health-compromising effects of considering oneself to have a low socioeconomic position. People with a low subjective socioeconomic status, who position themselves on the bottom tray of a presented societal ladder, have a higher risk for poor health outcomes compared to people positioning themselves on the top (Demakakos, Nazroo, Breeze, Marmot, 2008; Hamad, Fernald, Karlan \& Zinman, 2008; Lemeshow, Fisher, Goodman, Kawachi, Berkley et. al., 2008). Similarly, the literature on relative deprivation (in contrast to absolute deprivation), is primarily about processes of social comparison and its adverse health consequences in the case of downward comparisons (McEwen \& Gianoros, 2010). In our wealthy societies, that are increasingly based on a meritocratic ideology (Simons, Groffen \& Bosma, 2013), healthcompromising downward social comparisons underlying relative deprivation might become increasingly more important. Some have hypothesized that this psychosocial pathway via a downward social comparison results in a state of "internalized inferiority" (Marmot, 2004). To our knowledge, the hypothesis of internalized inferiority being more common in low socioeconomic status groups has not yet been examined explicitly.

Two concepts related to this internalized inferiority might be shame and social inadequacy (Gilbert, 2000). The state of internalized inferiority is mostly described as a cognitive negative comparison by an individual comparing himself with others who are socioeconomically better-off (Marmot, 2004; Gilbert, 2000). Environmental influences in low socioeconomic status groups may promote a state of submissiveness in these people. Fewer choices when buying basic and luxury goods, due to restricted financial resources, and work conditions characterized by low control, might be such environmental influences. Submissiveness, shame, and experienced social inadequacy might be embedded in the self-image of people from lower socioeconomic status groups (Gilbert, 2000). Shame is the feeling that one is negatively judged by others. Shame over longer periods has been found to increase the pro-inflammatory cytokine activity and cortisol levels, which might impact the immune system (Dickerson, Gruenewald, \& Kemeny, 2004). Shame is also 
associated with the development of depression (Orth, Berking \& Burkhardt, 2006). Social inadequacy is the anxiety for and when dealing with other people; people with a high social inadequacy are not comfortable in interacting with other people. Prolonged periods of experienced inadequacy lead to social deprivation (Greca \& Lopez, 1998) and symptoms of depression (Gilbert, 2000).

Using data from the Dutch SMILE study on middle-aged and older persons, we set out to examine whether socioeconomic status affects a person's perceived social inadequacy and shame. The educational and income-related components of socioeconomic position (from both childhood and adulthood) were compared regarding their independent influences.

\section{Method}

\section{Study population}

Data from the longitudinal Dutch SMILE (Studie naar Medische Informatie en Leefwijzen in Eindhoven) study were used. Between November 2002 and May 2010, there were annual questionnaires that were sent to 55 year old and older people in Eindhoven, in the south-eastern part of The Netherlands. In 2008, there were 3,774 respondents to the questionnaire including social inadequacy. Due to both attrition and new people continuously entering the cohort (when they move into the area or become 55 years old), excluding persons with missing values on any of the relevant variables for this study, led to a sample of 1,323 respondents (35\%). The average age was 70.27 years (SD $=7.31$ and range $=58$ to 94 ) and 47.4 percent were men. More background information on the SMILE study can be found elsewhere (Akker, van den et. al., 2008). The medical ethical committee of the Maastricht Academic Hospital approved the study protocol of the SMILE study.

\section{Measures}

\section{Internalised inferiority}

Social inadequacy was measured in 2008 by the social inadequacy subscale from the Dutch Personality Questionnaire (Luteijn, Starren \& Dijk van, 2000). One of the items was "I don't like talking to strangers". The sum score on the 15 items was computed resulting in a variable ranging from o to 30 (Cronbach's $\alpha=0.89$ ). Shame was measured in 2009 by the Differential Emotion Scale IV, Shame sub-scale, consisting of three items (Izard, 1991). One of the items is: "In your daily life, how often do you feel embarrassed when anybody sees you make a mistake?". Sum scores were computed resulting in a variable ranging from 3 to 15 (Cronbach's $a=0.77$ ). Both variables were dichotomised based upon the worst quintile. 


\section{Socioeconomic status in adulthood}

Income was measured in November 2002, May 2003 and May 2006 by questions on the persons' monthly household net income (in 11 categories). Income was recoded into a continuous equivalent income variable by using the midpoint income for each category and dividing the result by the square root of the number of people living from the household income (OECD, 2011). The mean income across the three measurement phases was computed. Education was measured by the mean of six measures of the educational level between 2002 and 2007 (seven ordinal categories). Both income and education were categorised into thirds using tertiles.

\section{Socioeconomic status in childhood}

Poverty in childhood was measured in May 2004 by asking people whether in childhood there was too little money to buy food or new clothes or shoes (responses ranging from 1. never to 5 . yes, continuously). Education of the father and mother was measured in May 2005 (seven ordinal categories). Father's and mother's educational level were combined choosing the highest level. Both poverty and educational level of the parents were categorised into thirds using tertiles.

\section{Analyses}

Logistic regression analyses were used to estimate the influence of socioeconomic indicators on reports of social inadequacy (2008 measure) and shame (2009). These analyses were controlled for age and sex (model 1); all four indicators were also controlled for each other (model 2).

\section{Results}

Of the persons reporting social inadequacy, 28.9 percent reported increased experiences of shame compared with 12.2 percent of those who did not report social inadequacy (not tabulated) $\left(p\left(c^{2} i^{2}\right)<0.001\right)$. Both adulthood income and educational level affected reports of social inadequacy (Table 1, model 2). Those with a low income or a low educational level had a 1.88 (95\% Cl: 1.23-2.88) and 1.58 (1.04-2.40) higher odds of social inadequacy reports compared with those who were better-off. Recalls of parents with a low educational level was also related to reports of social inadequacy, but this was fully mediated by adulthood socioeconomic circumstances (the odds ratio decreased from 1.41 to 0.98 ). Poverty in childhood was strongly associated with reports of shame ( $\mathrm{OR}=2.20 ; 95 \% \mathrm{Cl}: 1.39-3.48)$. The associations of adulthood income and education with shame lost their (marginal) statistical significance when controlled for the socioeconomic indicators in early life. 
Table 1. Odds ratios ( $95 \%$ confidence intervals) of social inadequacy and shame by socioeconomic indicators, adjusted for age and sex (model 1: socioeconomic indicators not controlled for each other and model 2: socioeconomic indicators controlled for each other).

\begin{tabular}{|c|c|c|c|c|c|c|c|}
\hline & \multirow[b]{2}{*}{$\mathrm{N}$} & \multicolumn{3}{|c|}{ Social inadequacy } & \multicolumn{3}{|c|}{ Shame } \\
\hline & & $\%$ & Model 1 & Model 2 & $\%$ & Model 1 & Model 2 \\
\hline \multicolumn{7}{|l|}{ income } & \\
\hline High & 464 & 13.1 & 1.00 & 1.00 & 12.1 & 1.00 & 1.00 \\
\hline Medium & 537 & 18.1 & $1.44(1.01-2.04)$ & $1.28(0.88-1.86)$ & 17.1 & $1.54(1.08-2.21)$ & $1.47(1.00-2.16)$ \\
\hline Low & 322 & 27.3 & $2.41(1.67-3.48)$ & $1.88(1.23-2.88)$ & 16.8 & $1.52(1.01-2.29)$ & $1.33(0.83-2.13)$ \\
\hline $\begin{array}{l}\text { Adulthood } \\
\text { education }\end{array}$ & & $*$ & & & & & \\
\hline High & 546 & 14.8 & 1.00 & 1.00 & 13.7 & 1.00 & 1.00 \\
\hline Medium & 368 & 14.9 & $1.01(0.69-1.48)$ & $0.88(0.59-1.33)$ & 14.4 & $1.05(0.71-1.55)$ & $0.94(0.62-1.43)$ \\
\hline Low & 409 & 26.9 & $2.09(1.50-2.91)$ & $1.58(1.04-2.40)$ & 18.1 & $1.42(0.99-2.04)$ & $1.18(0.75-1.86)$ \\
\hline $\begin{array}{l}\text { Childhood } \\
\text { poverty }\end{array}$ & \multicolumn{6}{|c|}{ poverty } & \\
\hline Low & 811 & 17.8 & 1.00 & 1.00 & 12.6 & 1.00 & 1.00 \\
\hline Medium & 380 & 19.5 & $1.13(0.83-1.55)$ & $1.03(0.75-1.42)$ & 17.6 & $1.50(1.07-2.10)$ & $1.46(1.04-2.05)$ \\
\hline High & 132 & 21.2 & $1.28(0.81-2.03)$ & $1.07(0.67-1.71)$ & 25.0 & $2.29(1.46-3.58)$ & $2.20(1.39-3.48)$ \\
\hline \multicolumn{8}{|l|}{$\begin{array}{l}\text { Childhood } \\
\text { education }\end{array}$} \\
\hline High & 344 & 15.1 & 1.00 & 1.00 & 14.5 & 1.00 & 1.00 \\
\hline Medium & 388 & 18.3 & $1.25(0.84-2.03)$ & $1.01(0.66-1.71)$ & 16.5 & $1.16(0.78-1.74)$ & $0.97(0.63-1.50)$ \\
\hline Low & 591 & 20.8 & $1.41(0.98-2.01)$ & $0.98(0.65-1.48)$ & 14.9 & 1.09 (0.74-1.59) & $0.84(0.55-1.30)$ \\
\hline
\end{tabular}

${ }^{*} \mathrm{p}\left(\mathrm{chi}^{2}\right)<0.05$.

Using the original continuous variables and linear regression analyses, a similar pattern of findings was found (not tabulated). The dose-response association between socioeconomic status and social inadequacy and shame was confirmed by the absence of significant quadratic terms of the continuous measures of socioeconomic status. Two-way interactions between the socioeconomic indicators, between age and the socioeconomic indicators, and between sex and the socioeconomic indicators were not statistically significant.

\section{Discussion}

In this sample of middle-aged and older Dutch men and women, we found that a person's socio-economic status in adulthood, especially the financial indicators, has a significant and independent influence on reports of social inadequacy. Poverty in childhood was 
related to feelings of general shame, independent of the other socioeconomic indicators. Earlier research already found that feelings of shame develop in childhood, which might explain why there was no additional effect on shame from socioeconomic circumstances in adulthood (Lewis, 1992; Wursmer, 1994). To our knowledge, this is the first study explicitly showing that reports of internalized inferiority are more common in lower socioeconomic status groups. Although associations with physical and mental health have still to be examined, our findings already suggest that the psychosocial mechanism via negative social comparisons and internalized inferiority might indeed contribute to the heightened risks of adverse health outcomes in people with a lower socioeconomic position (Gilbert, 2000).

Between-country differences in income inequality and the meritocratic ideology might moderate the effect of socioeconomic status on internalized inferiority. In countries where incomes are more equal, citizens might experience less feelings of internalized inferiority, because the referential edges for social comparison lay closer together in these countries (Wilkinson, 2006). Similarly, in countries where the meritocratic ideology is less strongly embedded in the sociocultural system, people with lower socioeconomic positions might also less often experience internalized inferiority, as the stigmatization of being at the bottom of the socioeconomic hierarchy might be less strong (Simons, Groffen \& Bosma, 2013). Further research might study the extent to which socioeconomic status internalized inferiority associations are indeed stronger in countries with more income inequality or a stronger meritocratic ideology.

Sapolsky (2004) found that primates, based on their physique, were divided into a social hierarchy. In primates that were categorized into a low social class, the fight-or-flight system was triggered (Sapolsky, 2004). Some primates who triggered the fight reaction became aggressive towards congeners, while others, triggering the flight reaction, became submissive. In both groups, stress-related neuroendocrine responses were found that might increase the risk of mental and physical health problems (Sapolsky, 2004). A similar mechanism might operate in humans. Low socioeconomic status, and especially financial indicators, could similarly trigger a fight-or-flight response, resulting in either aggression or submissiveness. However, using the same dataset of middle-aged and older persons, Klabbers and colleagues (2009) found that "rebelliousness" was rather uncommon. The flight reaction might thus be more common than the fight reaction in older age groups, leading to reactions in terms of submissiveness, social inadequacy, and shame. More research is needed into whether this response results in poor health outcomes via neuroendocrine pathways (Dickerson, Gruenewald, \& Kemeny, 2004; Gilbert, 2000).

Other research has found additional evidence for a reversed association between social inadequacy and income (Groffen, Bosma, Akker van den, Kempen \& Eijk van, 2009). They 
found support for social inadequacy affecting the risk of an income drop in older age. Hence, the mechanism could act as vicious circle, in which low income will result in high social inadequacy and high social inadequacy will result in lower income. It supports the hypothesis of individual differences (e.g. in personality and genetic make-up) being as important for life-course pathways as social differences (e.g. in socioeconomic status) (Mackenbach, 2005). Simultaneously, it points to the difficulties of interventions aimed at tackling socioeconomic differences in health. As reported above, both macro-economic and -cultural contexts (e.g. meritocracy) and individual differences might be addressed when breaking the vicious circle. On the individual level, one might think of intervention possibilities based on the possible associations of reports of inferiority with learned helplessness, low control beliefs, and depression.

Some limitations should be considered. First, this research only investigated adults aged 55 years and older. Further research should investigate the associations among a younger study population in which the meritocratic ideology and the increased norm of individual responsibility might be stronger. Second, in prior analyses we examined the influences on health-related functioning, but possibly as a result of only having two years of follow-up of health between 2008 and 2010, only minor longitudinal change and variation therein occurred. Third, the categorization into thirds or dichotomies was crude, but the linear regression with the continuous variables showing a similar pattern of findings was reassuring. Fourth, serious cases of social inadequacy and shame were scarce: 84.5 percent scored less than 2.5 on shame (range 1 to 5 ) and 83.5 percent scored one or less on social inadequacy (range o to 2). This implies that internalized inferiority, similar to "rebelliousness", was also relatively uncommon in this older group of people. More research is needed to find out whether extreme scores on any response variable are less common in older people (perhaps due to habituation). Fifth, non-response among all participants was relatively high. As a result there might be some risk for selection-bias, while questions about shame and social inadequacy might be avoided by people who experience a lot of shame and/ or social inadequacy. For this purpose a study among the non-responders can be recommended.

\section{Conclusion}

In this cohort of middle-aged and older men and women, socioeconomic status, especially its financial component, impacts cognitions and feelings of internalized inferiority. Childhood socioeconomic circumstances, particularly experiences of poverty, contributed independently. Our findings suggest that negative social comparisons and internalized inferiority might be possible key players in the association between low socioeconomic status and poor health. 


\section{References}

1. Akker, van den, M., Spigt, M.G., Raeve, de, L., Steenkiste, van, B., Metsemakers, J.F.M., Voorst, van, E.J. \& Vries, de, H. (2008). The SMILE study: a study of medical information and lifestyles in Eindhoven, the rationale and contents of a large prospective dynamic cohort study. BMC Public Health. 8:19.

2. Demakakos, P., Nazroo, J., Breeze, E. \& Marmot, M. (2008). Socioeconomic status and health: the role of subjective social status. Social Science \& Medicine. 67:330-340.

3. Dickerson, S.S., Gruenewald, T.L. \& Kemeny, M.E. (2004). When the social self is threatened: Shame, Physiology, and Health. Journal of Personality. 72(6): 1191-1216.

4. Gilbert, P. (2000). The relationship of shame, social anxiety and depression: the role of the evaluation of social rank. Clinical Psychology and Psychotherapy. 7: 174-189.

5. Greca La, A.M. \& Lopez, N. (1998) Social Anxiety among adolescents: Linkages with peer relations and friendship. Journal of Abnormal Child Psychology. 26(2): 83-94.

6. Groffen, D.A.I., Bosma, H., Akker van den, M., Kempen, G.I.J.M. \& Eijk van, J.Th.M. (2009). Personality and health as predictors of income decrease in old age: Findings from the longitudinal SMILE study. European Journal of Public Health. 19(4): 418-423.

7. Hamad, R., Fernald, L.C.H., Karlan, D.S. \& Zinman, J. (2008). Social and economic correlates of depressive symptoms and perceived stress in South African adults. Journal of Epidemiology and Community Health 62: $538-544$.

8. Izard, E. E. (1977). Human Emotions. New York: Plenum Press

9. Klabbers, G., Bosma, H., Akker van den, M., Boxtel van, M.P.J., Kempen, G.I.J.M., McDermott, M.R. \& Eijk van, J.TH.M. (2009) Measuring Rebelliousness and Predicting Health Behavior and Outcomes: An Investigation of the Construct Validity of the Social Reactivity Scale. Journal of Health Psychology. 14(6): 771-779.

10. Lemeshow, A.R., Fisher, L., Goodman, R., Kawachi, I., Berkey, C.S. \& Colditz, G.A. (2008). Subjective social status in the school and change in adiposity in female adolescents. Archives of Pediatrics \& Adolescent Medicine. 162: $23-28$.

11. Luteijn, F., Starren, J., \& Van Dijk, H. (2000) Handleiding Nederlandse Persoonlijkheids Vragenlijst (herziene uitgave). Lisse: Swets \& Zeitlinger.

12. Mackenbach, J.P. (2005). Genetics and health inequalities: hypotheses and controversies. J Epidemiol Community Health. 59: 268-273

13. Marmot, M.G. (2004). The status syndrome: How social standing affects our health and longevity. New York: Times Books.

14. McEwen, B.S., \& Gianaros, P.J. (2010). Central role of the brain in stress and adaption: links to socioeconomic status, health, and disease. Annals of the New York Academy of Sciences. 1186:190-222

15. OECD. (2011). Divided we stand. Why inequality keeps rising. OECD: OECD Publishing.

16. Orth, U., Berking, M. \& Burkhardt, S. (2006). Self-conscious Emotions and Depression: Rumination explains why shame but not guilt is maladaptive. Pers Soc Psychol Bull. 32(12): 1608-1619.

17. Sapolsky, R.M. (2004). Social Status and Health in Humans and Other Animals. Annual Review of Anthropology. 33: 393-418.

18. Simons, A.M.W., Groffen, D.A.I. \& Bosma, H. (2013) Income-related health inequalities: does perceived discrimination matter. International Journal of Public Health. 58(4): 513-520.

19. Wilkinson, R.G. (2006). Health, Hierarchy, and Social Anxiety. Annals of the New York Academy of Sciences. $896: 48-63$ 